\title{
The neglected role of abdominal compliance in organ-organ interactions
}

\author{
Manu L. N. G. Malbrain ${ }^{1 *}$, Yannick Peeters ${ }^{1}$ and Robert Wise ${ }^{2,3}$
}

\begin{abstract}
This article is one of ten reviews selected from the Annual Update in Intensive Care and Emergency medicine 2016. Other selected articles can be found online at http://www.biomedcentral.com/ collections/annualupdate2016. Further information about the Annual Update in Intensive Care and Emergency Medicine is available from http:// www.springer.com/series/8901.
\end{abstract}

\section{Background}

Over the last few decades, increasing attention has been given to understanding the pathophysiology, etiology, prognosis, and treatment of elevated intra-abdominal pressure (IAP) in trauma, surgical, and medical patients. However, there is still a relatively poor understanding of intra-abdominal volume (IAV) and the relationship between IAV and IAP (i.e., abdominal compliance $\left[C_{a b}\right]$ ). According to the consensus definitions proposed by the World Society on Abdominal Compartment Syndrome (WSACS), $C_{a b}$ is defined as a measure of the ease of abdominal expansion, determined by the elasticity of the abdominal wall and diaphragm [1]. $C_{a b}$ should be expressed as the change in IAV per change in IAP (expressed in $\mathrm{ml} / \mathrm{mmHg}$ ). $\mathrm{C}_{\mathrm{ab}}$ is one of the most neglected parameters in critically ill patients, despite playing a key-role in understanding the deleterious effects of unadapted IAV on IAP, organ-organ interactions and end-organ perfusion $[2,3]$. Although there are some papers related to $C_{a b}$ in surgical patients, only a few papers have been published addressing this issue in critically ill patients [2-4].

\footnotetext{
* Correspondence: manu.malbrain@skynet.be

${ }^{1}$ Ziekenhuis Netwerk Antwerpen, ZNA Stuivenberg, Intensive Care Unit and

High Care Burn Unit, B-2060 Antwerp, Belgium

Full list of author information is available at the end of the article
}

\section{Definitions}

The abdominal compartment

The abdominal compartment is a technical masterpiece as this small human cavity houses $8.5 \mathrm{~m}$ of intestine. Analogous to the skull, the abdomen can be considered as a relatively closed box with an anchorage above (costal arch) and rigid (spine and pelvis) or partially flexible walls (abdominal wall and diaphragm) filled with solid organs and hollow viscera [2]. The size and/or volume of the abdomen may be affected by the varying location of the diaphragm, the shifting position of the costal arch, the contractions of the abdominal wall, and the contents contained within the intestines.

\section{The abdominal wall}

The abdominal wall represents the boundaries of the abdominal cavity between the xyphoid bone and costal margins cranially and the iliac and pubic bones of the pelvis caudally. $C_{a b}$ is mainly defined by the elasticity of the different muscle layers of the abdominal wall (anterior and lateral parts) and to a lesser extent the diaphragm muscle.

\section{Intra-abdominal pressure and abdominal hypertension Intra-abdominal pressure}

The IAP is the steady-state pressure concealed within the abdominal cavity. The reference standard for intermittent IAP measurements is via the bladder. IAP should be expressed in $\mathrm{mmHg}$ and measured at the end of exhalation in the supine position after ensuring that abdominal muscle contractions are absent and with the transducer zeroed at the level where the midaxillary line crosses the iliac crest [1].

\section{Baseline IAP}

Also called resting, starting, static or opening IAP during laparoscopy, the baseline IAP is the IAP obtained at normal resting conditions [2]. Normal IAP is considered as $5-7 \mathrm{mmHg}$ in healthy individuals, and approximately $10 \mathrm{mmHg}$ in critically ill adults [5]. 


\section{Intra-abdominal hypertension (IAH)}

IAH is defined as a sustained or repeated pathological elevation in IAP $\geq 12 \mathrm{mmHg}$. IAH is graded as follows: Grade I, IAP 12-15 mmHg; Grade II, IAP 16$20 \mathrm{mmHg}$; Grade III, IAP 21-25 mmHg; and Grade IV, IAP $>25 \mathrm{mmHg}[1]$.

\section{Abdominal compartment syndrome (ACS)}

ACS is defined as a sustained IAP $>20 \mathrm{mmHg}$ (with or without an abdominal perfusion pressure [APP] $<60 \mathrm{mmHg}$ ) that is associated with new organ dysfunction/failure. In contrast to IAH, ACS is an all-or-nothing phenomenon [1].

\section{Delta IAP}

$\triangle \mathrm{IAP}$ is calculated as the difference between the endinspiratory $\left(\mathrm{IAP}_{\mathrm{ei}}\right)$ and the end-expiratory $\left(\mathrm{IAP}_{\mathrm{ee}}\right)$ IAP value. The higher the $\triangle \mathrm{IAP}$, the lower the $\mathrm{C}_{\mathrm{ab}}$.

$$
\Delta \mathrm{IAP}=\mathrm{IAP}_{\mathrm{ei}}-\mathrm{IAP}_{\mathrm{ee}}
$$

\section{Abdominal pressure variation (APV)}

$\mathrm{APV}$ is calculated as the difference between the $\mathrm{IAP}_{\mathrm{ei}}$ and the $\mathrm{IAP}_{\mathrm{ee}}$ value, or $\triangle \mathrm{IAP}$, divided by the mean IAP and is expressed as a percentage. The higher the APV, the lower the $\mathrm{C}_{\mathrm{ab}}$.

$$
A P V=\frac{\triangle I A P}{\text { mean IAP }}=\frac{I A P_{e i}-I A P_{e e}}{\text { mean IAP }}
$$

\section{Intra-abdominal volume}

\section{Baseline IAV}

Also called resting, starting or static IAV, the baseline IAV is the IAV at baseline conditions without additional pathologic volume increase or $\mathrm{C}_{\mathrm{ab}}$ decrease, with corresponding baseline IAP. The baseline IAV in healthy individuals is around 131 [2].

\section{Abdominal distension}

This is defined as a sagittal abdominal diameter (approximately at the level of the umbilicus) higher than the virtual line between the xiphoid and symphysis pubis.

\section{Abdominal workspace}

This is the additional IAV that can be added to the baseline IAV when IAP is limited to a certain pressure (e.g., $14 \mathrm{mmHg}$ during laparoscopic surgery). The normal workspace during laparoscopy ranges between 3 and 61 .

\section{Maximal stretched volume}

The maximal volume is calculated as the baseline $\mathrm{IAV}+$ the maximal workspace resulting in maximal stretch of the abdominal cavity (from ellipse to sphere on a transverse plane). The maximal stretched volume depends on baseline IAP and $\mathrm{C}_{\mathrm{ab}}$.

\section{Abdominal compliance Abdominal compliance}

$\mathrm{C}_{\mathrm{ab}}$ is defined as the ease with which abdominal expansion can occur, and is determined by the elasticity of the abdominal wall and diaphragm. Increased compliance indicates a loss of elastic recoil of the abdominal wall. Decreased compliance means that the same change in IAV will result in a greater change in IAP. It should be expressed as the change in IAV per change in IAP $(\mathrm{ml} / \mathrm{mmHg})$ [1]. Normal $\mathrm{C}_{\mathrm{ab}}$ is around 250 to $450 \mathrm{ml} / \mathrm{mmHg}$.

\section{Abdominal PV relationship}

Importantly, $\mathrm{C}_{\mathrm{ab}}$ is measured differently than IAP, it is only a part of the total abdominal pressure-volume (PV) relationship.

$$
\begin{aligned}
& \text { Compliance }(\mathrm{C})=\frac{\Delta \mathrm{V}}{\Delta \mathrm{P}} \text { or thus } \mathrm{C}_{\mathrm{ab}}=\frac{\Delta \mathrm{IAV}}{\Delta \mathrm{IAP}} \\
& \text { Elastance }(\mathrm{E})=\frac{\Delta \mathrm{P}}{\Delta \mathrm{V}}=\frac{1}{\mathrm{C}} \text { or thus } \mathrm{E}_{\mathrm{ab}}=\frac{\Delta \mathrm{IAP}}{\Delta \mathrm{IAV}}
\end{aligned}
$$

The relationship between IAV and IAP is curvilinear with an initial linear part followed by an exponential increase once a critical volume is reached (Fig. 1).

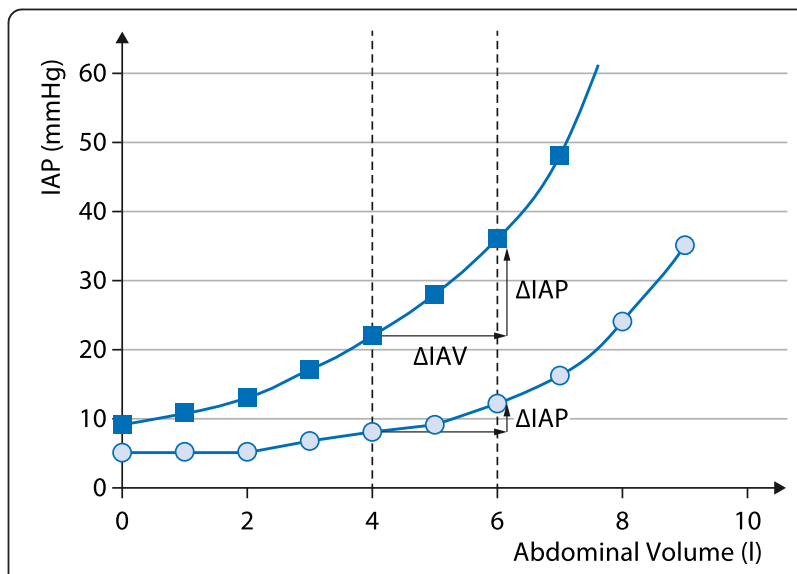

Fig. 1 Pressure-volume curve in the abdominal compartment. Abdominal pressure-volume curves in a patient with low abdominal compliance (squares) and normal compliance (circles). At a baseline IAV of $4 \mathrm{I}$, the same $2 \mathrm{I}$ increase in IAV will only lead to a small increase in IAP $(5 \mathrm{mmHg}$ ) in a patient with good abdominal compliance versus a high increase in IAP $(15 \mathrm{mmHg})$ in the case of a stiff abdominal wall and diaphragm. The compliance is $133 \mathrm{ml} / \mathrm{mmHg}$ [2000/(37 - 22)] versus $400 \mathrm{ml} / \mathrm{mmHg}[2000 /(12-7)]$ for the same change in IAV from 4 to 6 I. Adapted from [2] with permission 


\section{Pathophysiology}

\section{Accommodation of the abdominal cavity}

In contrast to the intracranial compartment that is confined within a rigid bony structure, the abdominal compartment can change shape during increasing IAV. During the initial phase of increasing IAV (e.g., laparoscopic insufflation), IAP only rises minimally (linear 'reshaping phase' from spherical to circular shape). This is followed by a 'stretching phase' of the rectus abdominis muscle (curvilinear phase) and finally, when further IAV is added, only small increases in IAV will result in dramatic increase in IAP (exponential 'pressurization phase') (Fig. 2) [6, 7]. During the stretching phase, the shape of the abdomen will change from elliptical to spherical. This change in shape is mainly due to an increase in the antero-posterior diameter and a decrease in the transverse diameter (transverse plane) of the internal abdominal perimeter [8-12].

\section{Predictors for stretching and reshaping capacity}

Factors determining the reshaping properties of the abdominal wall and diaphragm are not well understood

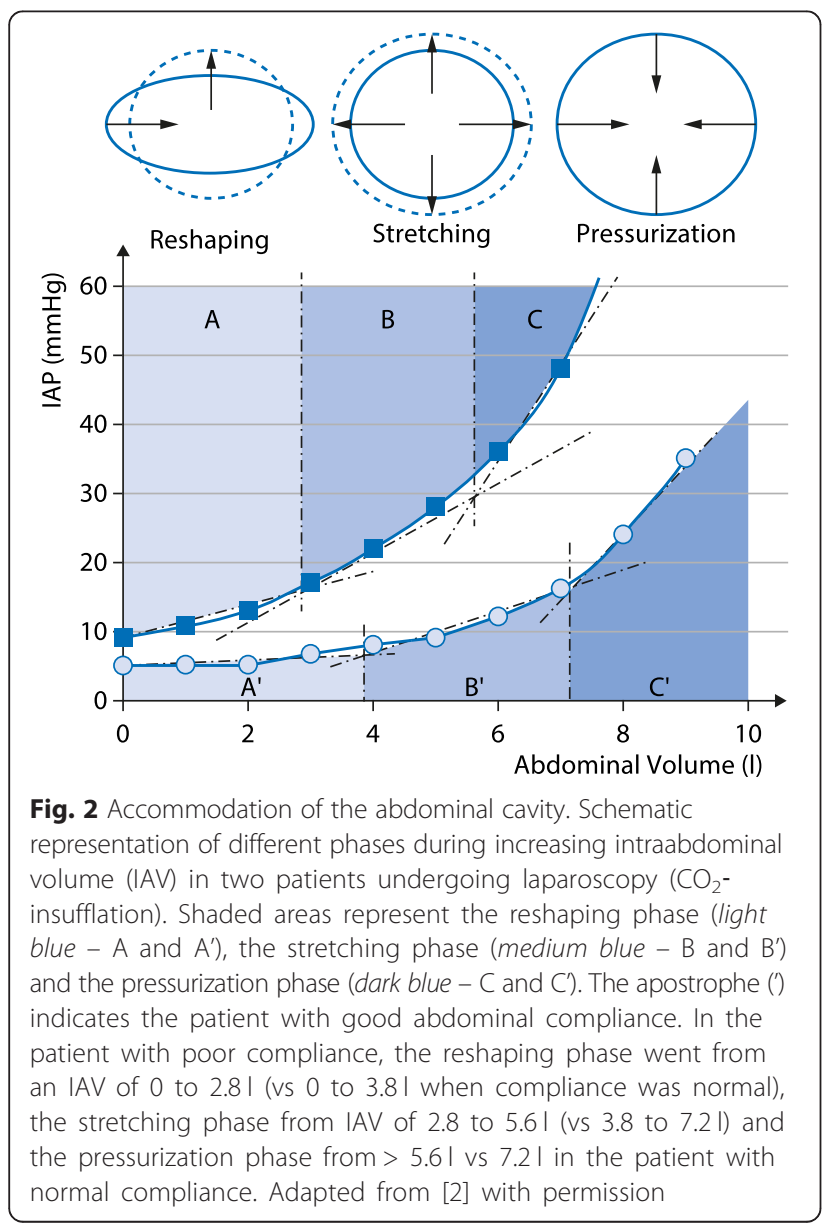

but the mechanical properties are related to $C_{a b}$. The stretching capacity is influenced by body anthropomorphy (weight, height, body mass index [BMI]), age, sex and visceral versus subcutaneous fat distribution [11]. Comorbidities like chronic obstructive lung disease (COPD) with emphysema (flatting of diaphragm), fluid overload (tissue and interstitial edema) or burn injury (with circular eschars) all have negative effects on stretching capacity. Android obesity usually results in increased visceral fat and a sphere-like baseline shape of the abdominal cavity with poor stretching capacity, whereas gynoid obesity presents with more subcutaneous fat for the same BMI or abdominal perimeter (Fig. 3). In gynoid obesity, the internal abdominal perimeter is elliptical. Patients with an ellipse-shaped internal perimeter have a much greater stretching capacity (and thus very good $\mathrm{C}_{\mathrm{ab}}$ ).

\section{Abdominal pressure-volume relationship}

A linear abdominal PV relationship has been described previously. However, this was mainly in studies where the observed IAP values were $<15 \mathrm{mmHg}$. During laparoscopy with limitation of insufflation pressures at 12 to $15 \mathrm{mmHg}$, the IAV did not reach a critical point at which an exponential increase in IAP occured [13]. As discussed above, the initial phase of the PV curve may indeed be linear (as observed during laparoscopy) but the remaining part is curvilinear or rather exponential [13-15]. Because of this exponential relationship, it is important to know both the shape and the position on the curve, as the actual position will determine the corresponding $\mathrm{C}_{\mathrm{ab}}$. In patients with IAH a small increase in IAV may push them into ACS (especially if $C_{a b}$ is low) and, vice versa, in patients with ACS a small decrease in IAV (with paracentesis) may result in a dramatic improvement in IAP.

\section{Measurement}

Intra-abdominal pressure

Because of the fluid-like nature of the abdomen, following Pascal's law, the IAP can be measured in nearly every part of it. Rectal, uterine, inferior vena cava, bladder and gastric pressure measurements have all been described [16]. The use of direct intraperitoneal pressure measurement cannot be advocated in patients because of the complication risks, such as bleeding or infection. Bladder pressure measurements have been forwarded as the gold standard with the technique suggested in the WSACS consensus guidelines [1].

\section{Intra-abdominal volume}

The abdominal volume is more difficult to measure. However, it can be estimated by anthropomorphic 


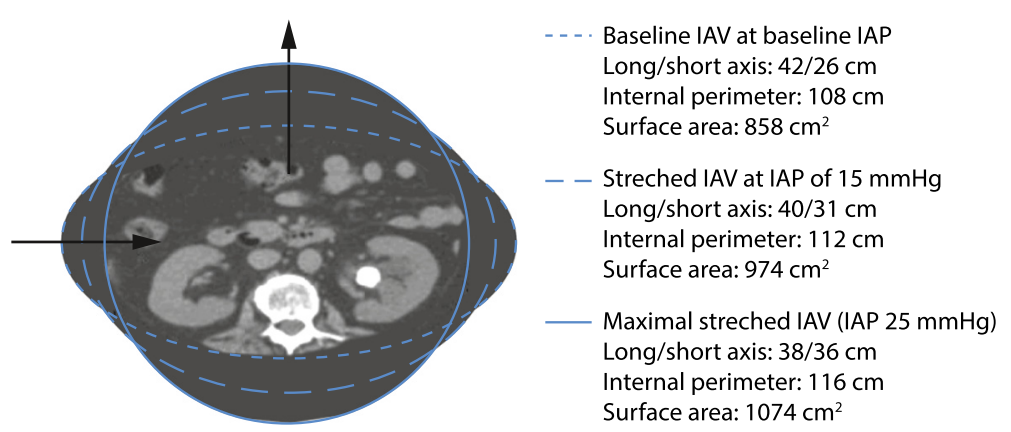

Fig. 3 Evolution of internal abdominal cavity perimeter during increase in volume. In case of gynoid obesity, the internal abdominal perimeter is shaped as an ellipse. Patients with an ellipse-shaped internal perimeter have a huge stretching capacity (and thus very good abdominal compliance); this is illustrated with the progression of the shape from ellipse (dotted line) at baseline to a sphere (solid line) at very high intra-abdominal pressures (IAP) obtained during laparoscopy. The arrows show the centripetal movement of the lateral edges of the ellipse and a centrifugal movement of the cranio-caudal edges. During increase in intra-abdominal volume (IAV) from baseline to stretched and maximal stretched IAV, the difference between the long and short axes of the ellipse decreases, while the internal perimeter and surface area increase. At maximal stretch, the external and internal abdominal perimeter are equal. Patients with android obesity do not have this reshaping and stretching capability.

Adapted from [2] with permission

indices and imaging techniques. Anthropomorphicbased indices for estimation of IAV have been described in obesity [17]. However, BMI does not correlate with $\mathrm{C}_{\mathrm{ab}}$ but does correlate with IAP at the resting volume. This only applies in healthy individuals and sometimes in critically ill patients. The external abdominal perimeter (or circumference), although often used in the past, correlates reasonably with IAV, but poorly with IAP [18]. Changes in external abdominal perimeter over time on the other hand may correlate well with changes in IAP [18]. Another useful parameter is the waist-to-hip ratio. The waist is the smallest horizontal girth between the rib cage and iliac crest and the hip is the largest horizontal girth between waist and thigh. The waist-to-hip ratio correlates with IAP in men only [17]. A promising index is the abdominal volume index (AVI). A formula developed for calculating AVI estimates the overall abdominal volume between the symphysis pubis and the xiphoid process. This measure theoretically includes intra-abdominal fat and adipose volumes, with the waist and the hip dimensions. Although this index is superior to BMI, waist-to-hip ratio, and waist circumference, it has not yet been correlated to IAP [19]. Recently, techniques for estimating abdominal volume via three-dimensional (3D) ultrasound, water-suppressed breath hold magnetic resonance imaging (MRI), and computed tomography (CT) have been described. These techniques have not yet gained entrance to the intensive care unit (ICU). Although 3D ultrasound cannot measure IAV in toto, it estimates the volumes of separate intra-abdominal organs. MRI and CT techniques calculate the visceral and subcutaneous fat volume or thus the volume of the adipose tissue. Quantitative CT analysis assessing volume, density and weight of abdominal organs may be a promising tool for the future $[9,10,20,21]$.

\section{Abdominal compliance \\ Qualitative measurement of abdominal wall tension during palpation}

The grade of indentation at the site where the downward force is applied can be measured during palpation of the abdomen. Palpation examines intra-abdominal tension, passive and active muscle tension. However, it is not able to quantify $\mathrm{C}_{\mathrm{ab}}$ properly nor has it been validated in the clinical setting. The use of an abdominal tensiometer has also been described; however, this technique is only in its infancy [22].

\section{PV relationship during laparoscopy with $\mathrm{CO}_{2}$ pneumoperitoneum}

It has been observed that the compliance of the abdominal cavity decreases when additional volume is added [23]. The linear abdominal PV curve changed to a rather exponential shape when a pressure of $15 \mathrm{mmHg}$ was achieved by insufflation of $\mathrm{CO}_{2}[13,14]$. In studies, the initial $\mathrm{C}_{\mathrm{ab}}$ at the beginning of the $\mathrm{CO}_{2}$ inflation varied between 333 and $400 \mathrm{ml} / \mathrm{mmHg}$ and at higher IAV (with corresponding IAP $>15 \mathrm{mmHg}$ ), the $\mathrm{C}_{\mathrm{ab}}$ decreased to 60 and $90 \mathrm{ml} / \mathrm{mmHg}$ [3]. Similar relationships have been described with addition or removal of gastric contents [3].

\section{PV relationship during drainage or addition of abdominal free fluid}

Measurements of $\mathrm{C}_{\mathrm{ab}}$ have been performed in humans by IAP assessment with at least two corresponding IAV values by addition of abdominal fluid during peritoneal 
dialysis or by drainage of intra-abdominal fluid (ascites in liver cirrhosis, peripancreatic fluid or pseudocyst, serous fluid collections in trauma or burns) [2-4].

\section{Interactions between different compartments Polycompartment model}

Being linked and bound by the diaphragm, the thoracic and abdominal compartments cannot be treated in isolation. Applied airway pressure $\left(\mathrm{P}_{\mathrm{aw}}\right)$ by mechanical ventilation will be transmitted to the lungs, pleural $\left(\mathrm{P}_{\mathrm{pl}}\right)$ and abdominal spaces (IAP). In a simplified model, the lung and thorax are in series and coupled to the diaphragm and abdomen in series. Changes in IAP are paralleled by changes in $\mathrm{P}_{\mathrm{pl}}$. Changes in thoracic compliance will be reflected by changes in abdominal compliance and vice versa; as a consequence, increased IAP will result in reduced chest wall compliance. The interactions between different compartments have been referred to as the polycompartment model and syndrome [24, 25]. For example, transmission of airway pressures to the abdomen results from interactions between the thoracic and abdominal compartment and the percentage of pressure transmission is called the thoraco-abdominal index (TAI) of transmission. This occurs in patients receiving positive pressure ventilation, application of positive end-expiratory pressure (PEEP), presence of intrinsic or auto-PEEP or a tension pneumothorax. Conversely, transmission of pressure from the abdomen to the thorax is called the abdomino-thoracic index (ATI) and occurs in any physiologic (pregnancy) or pathologic condition associated with increased IAP; the ATI ranges from 20 to $80 \%$ and is on average $50 \%$ $[26,27]$. The effects of increased IAP on end-organ function are numerous: neurologic, respiratory, cardiovascular and renal adverse effects have all been described in patients with IAH and ACS. Increased IAP leads to diminished venous return, necessitating more fluid loading, causing mesenteric vein compression and venous hypertension, finally triggering a vicious cycle.

\section{Estimation of abdominal compliance during low flow pressure volume loop}

$\mathrm{C}_{\mathrm{ab}}$ can be estimated by analysis of the dynamic changes caused by mechanical ventilation on IAP. During a low flow PV loop to determine the best PEEP, one can observe the change in mean IAP. The compliance obtained by this maneuver can be calculated as follows:

$$
\mathrm{C}_{\mathrm{abPV}}=\Delta \mathrm{V}_{\mathrm{T}} / \Delta \text { mean IAP }
$$

with $\Delta \mathrm{V}_{\mathrm{T}}$ the insufflated tidal volume and $\Delta \mathrm{IAP}$ the difference between meanIAP at the end and start of the PV loop.

\section{Estimation of abdominal compliance during mechanical ventilation}

Whilst looking at the effects of tidal volume excursions on IAP and by calculating the difference between $\mathrm{IAP}_{\mathrm{ei}}$ and $\mathrm{IAP}_{\mathrm{ee}}$, one can also obtain an idea of $\mathrm{C}_{\mathrm{ab}}[28]$ :

$$
\mathrm{C}_{\mathrm{abTV}}=\mathrm{V}_{\mathrm{T}} / \Delta \mathrm{IAP}
$$

The higher the respiratory excursions seen in a continuous IAP tracing, the lower the $C_{a b}$ (for the same tidal volume). Alternatively, the higher the IAP, the higher the $\triangle$ IAP or the lower the $C_{a b}$.

\section{Calculation of abdominal pressure variation}

As discussed previously, the higher the APV for any given IAP, the lower the $C_{a b}$ and vice versa, the lower the $C_{a b}$, the higher the APV; hence APV can be used as a non-invasive and continuous estimation of $C_{a b}$.

\section{Respiratory abdominal variation test (RAVT)}

A final non-invasive method for estimation of $\mathrm{C}_{\mathrm{ab}}$ can be done by performing a respiratory abdominal variation test (RAVT) (Fig. 4). The $\mathrm{C}_{\mathrm{ab}}$ obtained with RAVT correlates with the $C_{a b}$ obtained from $\triangle I A P$ during

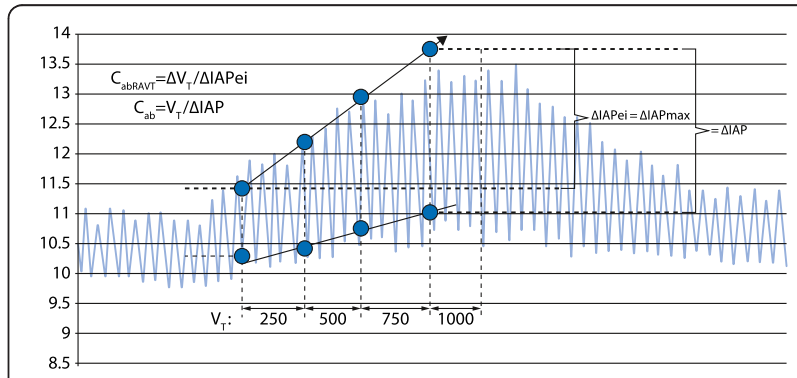

Fig. 4 Estimation of abdominal compliance $\left(C_{a b}\right)$ during the respiratory abdominal variation test (RAVT) in intermittent positive pressure ventilation (IPPV)-mode. The graph shows the smoothed average of a continuous intra-abdominal pressure (IAP) tracing (CiMON, Pulsion Medical System, Munich, Germany) obtained during the RAVT in IPPV mode. The tidal volume $\left(V_{T}\right)$ was increased stepwise from 250 to $1000 \mathrm{ml}$ with increments of $250 \mathrm{ml}$. At each $V_{T}$, the following parameters were recorded: end-expiratory IAP (IAP $\left.{ }_{e}\right)$, end-inspiratory IAP $\left(I_{A} P_{\mathrm{e}}\right)$, IAP and $\triangle I A P$. With increasing $V_{T}$ mainly the $I A P_{\text {ei }}$ increases whereas IAP ee remains relatively unchanged. During the RAVT, the diaphragm is displaced caudally and an additional volume is added to the abdominal cavity. The $\triangle I A V$ is probably correlated to the $\Delta V_{T}$ observed between the start and the end of the RAVT $(=750 \mathrm{ml})$. The slope of the curve connecting the $\mathrm{IAP}_{\mathrm{ei}}$ at each $V_{T}$ can be used to estimate the $C_{a b}$. The $C_{a b R A V T}$ in the sample shown can be calculated as follows: $C_{a b R A V T}=\Delta V_{T} / \Delta I A P_{e i}=750 /$ $(13.6-11.5)=357.1 \mathrm{ml} / \mathrm{mmHg}$ and this correlates well with the $C_{a b V T}: C_{a b V T}=V_{T} / \Delta I A P=1000 /(13.6-11)=384.6 \mathrm{ml} / \mathrm{mmHg}$. Adapted from [3] with permission 
mechanical ventilation; increasing tidal volume increases IAP $_{\text {ei }}$ while increasing PEEP increases IAP $_{\text {ee }}$.

$$
\mathrm{C}_{\mathrm{abRAVT}}=\Delta \mathrm{V}_{\mathrm{T}} / \Delta \mathrm{IAP}_{\mathrm{ei}}
$$

\section{Prognostic and predictive factors related to abdominal compliance}

Theoretically, $\mathrm{C}_{\mathrm{ab}}$ allows prediction of complications during laparoscopy and mechanical ventilation, identification of patients who would benefit from delayed abdominal closure, those in whom to monitor IAP, and those at risk during prone ventilation, etc. ... Therefore, prediction of poor or high $\mathrm{C}_{\mathrm{ab}}$ can be clinically important.

\section{Conditions associated with decreased abdominal compliance}

Aside from risk factors for IAH, patients should also be screened for risk factors for decreased $C_{a b}$. These are listed in Table 1 and can be divided into those related to body habitus and anthropomorphy; those related to comorbidities and/or increased non-compressible IAV; and those related to the abdominal wall and diaphragm [3].

Morbidly obese patients have a higher baseline IAP around $12-14 \mathrm{mmHg}$, and this is mainly related to the presence of central obesity [17, 29-32]. Morbidly obese patients with an android (mainly visceral and sphere shaped) fat distribution have a limited reserve to accommodate excess IAV than those patients who, for a similar BMI or abdominal perimeter, have a gynoid (mainly subcutaneous and ellipse shaped) fat distribution [17, 29]. On the other hand, subcutaneous fat accumulation may have a negative effect on the elastic properties of the abdominal wall, although the thin muscle layer may have a beneficial effect. Therefore, it is not possible to predict $\mathrm{C}_{\mathrm{ab}}$ in obese patients; in general $\mathrm{C}_{\mathrm{ab}}$ is decreased because of the increased baseline IAV.

\section{Conditions associated with increased abdominal compliance}

These are listed in Table 2 and can be divided into those related to body habitus and anthropomorphy; those related to absence of comorbidities and/or increased compressible IAV; and those related to abdominal wall and diaphragm. Chronic conditions will have higher $C_{a b}$ for the same change in IAV as illustrated in Fig. 5.

Previous stretching of the abdominal fascia increases $\mathrm{C}_{\mathrm{ab}}$. This can be explained by a gradual prestretching of the internal abdominal cavity perimeter during acute or progressive increased IAV (as is the case during laparoscopy, with pregnancy, peritoneal dialysis, cirrhotic ascites) $[7-9,33,34]$, which leads to increased reshaping capacity. Prestretching or overdistension may indeed
Table 1 Factors associated with decreased abdominal compliance. Adapted from [2] with permission

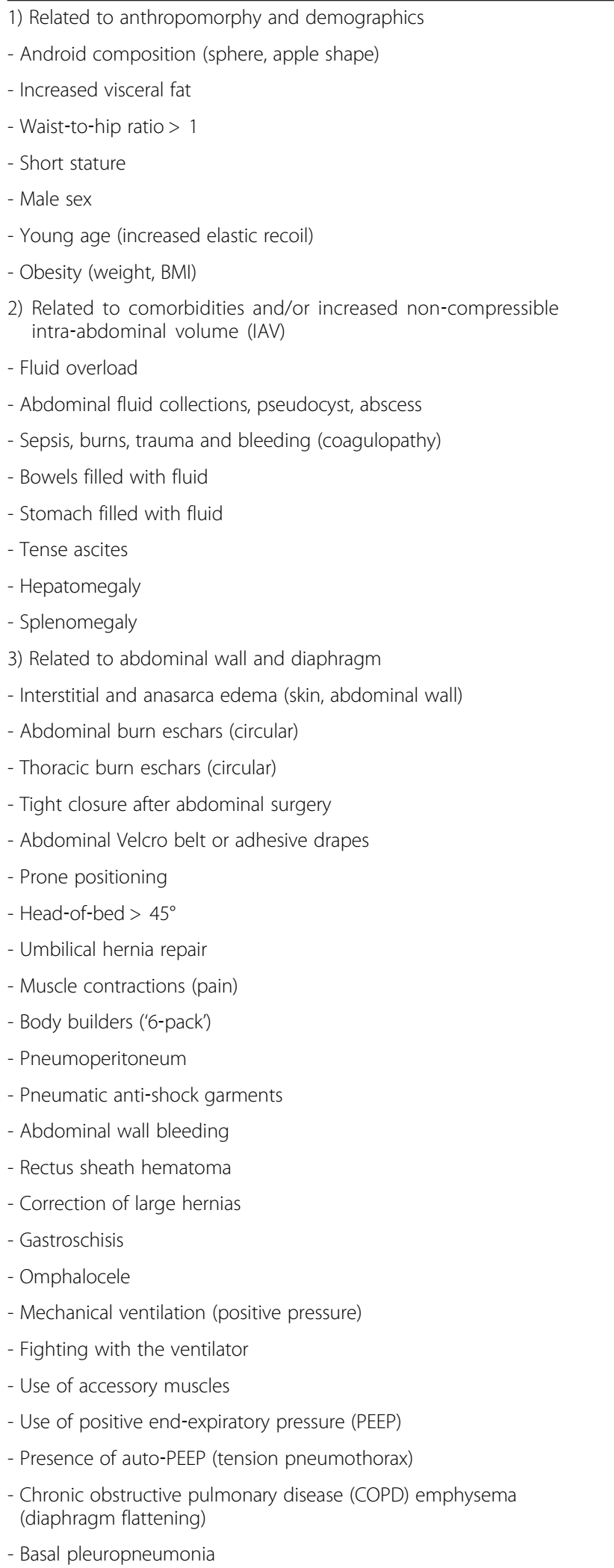


Table 2 Factors associated with increased abdominal compliance. Adapted from [2] with permission

1) Related to anthropomorphy and demographics
- Gynoid composition (ellipse, pear-shaped)
- Waist-to-hip ratio < 0.8
- Peripheral obesity
- Preferentially subcutaneous fat
- Height (tall stature)
- Old age (loss of elastic recoil)
- Female sex
- Lean and slim body
- Normal BMI
2) Related to absence of comorbidities and/or increased compressible
intra-abdominal volume (IAV)
- Absence of deadly triad: normothermia, normal pH, normal
coagulation
- Bowels filled with air
- Stomach filled with air
- Absence of fluid overload (second or third space fluid accumulation)
- Re Related to abdominal wall and diaphragm
- Previous pregnancy
- -
- Previous laparoscopy
- Previous abdominal surgery
- Abdominal wall lift
- Weight loss
- - Chronic intra-abdominal hypertension (IAH)
- Umbilical hernia (before repair)
- Burn escharotomy (thorax and/or abdomen)
-

result in tissue damage and fibrosis of the abdominal wall structure with lengthened muscle fibers and diminished elastic retraction capacity. History of a previous laparotomy may lead to scarring of the abdominal wall, which in combination with adhesions may cause decreased elasticity [33]. The $C_{a b}$ may be decreased or increased and the effect of previous laparotomy on baseline IAV and IAP is unpredictable. The use of external bandages (drapings, Velcro belt, etc.) or tight surgical closure causes a mechanical limitation; these should be

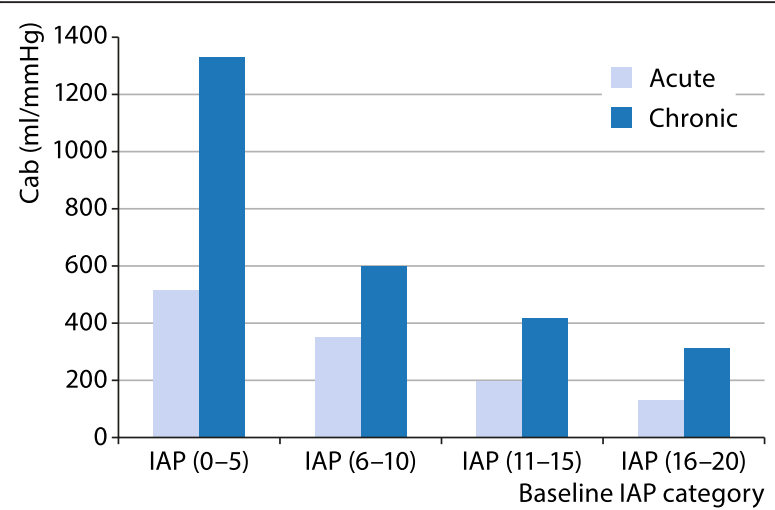

Fig. 5 Abdominal compliance $\left(C_{a b}\right)$ in relation to baseline intraabdominal pressure (IAP). Bar graph showing mean values of $C_{a b}$ $(\mathrm{ml} / \mathrm{mmHg}$ ) per baseline IAP category $(\mathrm{mmHg})$ in acute (light blue bars) and chronic (dark blue bars) conditions. Acute conditions are laparoscopy and evacuation of ascites, collections or hematomas in acutely ill patients, whereas chronic condition refers to peritoneal dialysis. Adapted from [3] with permission

avoided in high-risk patients and IAP should be measured during their use. In case of capillary leak, fluid overload and fluid collections, IAV and IAP will both increase while reshaping capacity and wall compliance will decrease.

\section{Treatment}

\section{How to decrease baseline IAP?}

In simple terms, in order to reduce IAP, either (additional) IAV has to be removed intra-luminally or intra-abdominally (e.g., weight loss, fluid removal via dialysis, ascites drainage, gastric suctioning, evacuation of abscess or hematoma, etc.), or the $C_{a b}$ has to be improved by increasing the internal abdominal cavity perimeter and surface area (pre-stretching, open abdomen treatment) $[35,36]$. Weight loss and the resulting decrease in BMI will decrease IAP [37].

\section{How to reduce IAV?}

The evacuation of intra-luminal and intra-abdominal contents can be done, for example, via placement of a nasogastric tube with suctioning with or without gastroprokinetics (cisapride, metoclopramide or erythromycin). Paracentesis with evacuation of ascites and the placement of a rectal tube in conjunction with enemas and colonoprokinetics (prostygmin) may also reduce IAV [38]. Colonic pseudo-obstruction or Ogilvie's syndrome may be treated with endoscopic decompression of large bowel or a surgical colostomy or ileostomy together with colonoprokinetics. When in doubt, imaging should be performed and ultrasound or CT guided drainage should be attempted in case of hematoma, abscess, fluid collections, etc. The correction of capillary leak and avoiding a 
positive fluid balance will eventually lead to a decreased IAV by decreasing organ and bowel edema [39]. This can be achieved with (hypertonic) albumin in combination with diuretics (furosemide), correction of capillary leak (antibiotics, source control, ...), the use of colloids instead of crystalloids and eventually dialysis or continuous veno-venous hemofiltration $(\mathrm{CVVH})$ with ultrafiltration [40]. Targeted APP with the use of vasopressors will reduce venocongestion and this will lower IAV (in analogy to the effect of norepinephrine on intracranial pressure and cerebral perfusion pressure) and dobutamine (but not dopamine) will improve splanchnic perfusion. Ascorbinic acid has been associated with a reduced incidence of secondary ACS in burn patients, although its routine use has yet to be validated.

\section{How to improve $\mathrm{C}_{\mathrm{ab}}$ ?}

Improvement in $\mathrm{C}_{\mathrm{ab}}$ should be performed in a stepwise approach as suggested by the WSACS consensus recommendations $[1,3]$.

\section{First step: ensure adequate sedation and analgesia}

Fentanyl should not be used as it may increase abdominal muscle tone while dexmedetomedine has superior effects over propofol. Thoracic epidural anesthesia has been shown to reduce IAP via an increase in $C_{a b}$ [41].

\section{Second step: remove constrictive bandages and eschars}

Any tight abdominal closure, like a Velcro belt to prevent incisional hernia in a patient with abdominal hypertension and end-organ dysfunction, should be removed immediately. Likewise, escharotomies (abdominal but also thoracic) will increase $C_{a b}$ while sternotomy will increase not only thoracic wall compliance but also $\mathrm{C}_{\mathrm{ab}}$ [42-44]. Placing a chest tube in case of a tension pneumothorax or pleural effusion will also increase $\mathrm{C}_{\mathrm{ab}}$.

\section{Third step: avoid prone and head of bed $>30^{\circ}$ and consider reverse Trendelenburg position}

Body positioning, such as the Trendelenburg position, may lower bladder pressure; however, it may also compromise respiratory function [29]. The use of head-ofbed elevation $>30^{\circ}$ may on the other hand increase bladder pressure and the head-of-bed $45^{\circ}$ position will increase IAP by 5 to $15 \mathrm{mmHg}$ [29]. Therefore, in patients with respiratory insufficiency who are mechanically ventilated, the anti-Trendelenburg position may be best to allow lung recruitment, oxygenation and ventilation [16]. During prone positioning there is merit in unloading the abdomen (abdominal suspension) as this will result in a decrease in chest wall compliance, while the effect of gravity will improve $C_{a b}$ and decrease IAP. During laparoscopy, body position can also help to optimize the laparoscopic workspace IAV. The Trendelenburg position with head-of-bed at $20^{\circ}$ provides the optimal workspace in lower abdominal laparoscopic surgery, while during upper abdominal laparoscopic surgery in obese patients, the beach-chair position (flexing the legs in reverse Trendelenburg) is optimal [45]. Laparoscopic insufflation pressures should at all times be limited to $15 \mathrm{mmHg}$. Higher working pressures cannot be routinely recommended in obese patients with high baseline IAP and in morbidly obese patients, open surgery seems the best option because of the high complication risk associated with pneumoperitoneum [31].

\section{Fourth step: lose weight and avoid fluid overload}

Similar to weight loss, avoiding a positive cumulative fluid balance and obtaining a negative fluid balance with the use of diuretics in combination or not with hypertonic solutions (albumin $20 \%$ ) [40, 46] will decrease interstitial edema of the abdominal wall and increase $\mathrm{C}_{\mathrm{ab}}$. Fluid resuscitation should be guided by volumetric (and not barometric) preload indicators and, if central venous pressure $(\mathrm{CVP})$ is used, transmural pressures should be calculated:

$$
\mathrm{CVP}_{\mathrm{tm}}=\mathrm{CVP}_{\mathrm{ee}}-\mathrm{IAP} / 2
$$

In case diuretics do not have a sufficient effect, renal replacement therapy with hemodialysis or $\mathrm{CVVH}$ can be used $[1,3]$.

\section{Fifth step: use neuromuscular blockers}

Theoretically, the use of neuromuscular blockade should not only lower baseline IAP but also improve $C_{a b}[1,3]$. However, some studies showed no additional increase in $\mathrm{C}_{\mathrm{ab}}$ after full block of abdominal muscle contractions (guided by train of four) [7].

\section{Sixth step: less invasive surgery}

Recently a less invasive percutaneous endoscopic abdominal wall component separation (EACS) technique has been described [47]. With this technique, the abdominal capacity (maximal stretched volume) increased by 11 while IAP decreased from $15.9 \pm 2.1$ to $11 \pm 1.5 \mathrm{mmHg}(\mathrm{p}<0.001)$ [47]. Another alternative for midline laparotomy is subcutaneous linea alba fasciotomy (SLAF), which seems a promising approach especially in secondary IAH and ACS [48].

When all the above listed treatment options fail to provide a sufficient decrease in IAP and IAV, the only definite solution is to perform a decompressive laparotomy that will assist with IAP, IAV and $C_{a b}$ [49]. 


\section{Conclusion}

$\mathrm{C}_{\mathrm{ab}}$ is a measure of the ease of abdominal expansion, determined by the elasticity of the abdominal wall and diaphragm. It is expressed as the change in IAV per change in IAP $(\mathrm{ml} / \mathrm{mmHg})$. The $\mathrm{C}_{\mathrm{ab}}$ baseline in 'resting conditions' is determined by the baseline IAP and IAV, the external and internal abdominal cavity perimeter and surface area and shape, the additional and maximal stretched volume, the presence of predisposing conditions and comorbidities as well as tissue properties of the fascia, abdominal wall and diaphragm. As such, $\mathrm{C}_{\mathrm{ab}}$ should be viewed separately from the abdominal wall and diaphragm compliance with its own specific elastic properties. $\mathrm{C}_{\mathrm{ab}}$ can be estimated based on demographic and anthropomorphic data and can be assessed by PV relationship analysis of the observed changes in IAP (mirroring induced changes in IAV). The abdominal PV relationship is believed to be linear up to pressures of $12-15 \mathrm{mmHg}$ and increases exponentially thereafter. $\mathrm{C}_{\mathrm{ab}}$ can also be estimated non-invasively by examining the interactions between pressure variations in the thorax and abdominal compartment during positive pressure ventilation. $\mathrm{C}_{\mathrm{ab}}$ is one of the most neglected parameters in critically ill patients, although it plays a key-role in understanding organ-organ interactions and the deleterious effects of unadapted IAV on IAP and end-organ perfusion. A large overlap exists between the treatment of patients with IAH and those with low $C_{a b}$, but when we identify the latter, we should potentially be able to anticipate and select the most appropriate medical or surgical treatment to avoid complications related to IAH or ACS.

\section{Competing interests}

Dr Manu Malbrain is member of the Executive Committee of the World Society of the Abdominal Compartment (WSACS) and current Treasurer; he is member of the medical advisory board of Pulsion Medical Systems (Maquet Getinge group). The other authors have no possible conflicts of interest related to the content of this paper.

\section{Authors' contributions}

All authors read and approved the final manuscript.

\section{Declarations}

Open access publication of this article was funded by the corresponding author.

\section{Author details}

${ }^{1}$ Ziekenhuis Netwerk Antwerpen, ZNA Stuivenberg, Intensive Care Unit and High Care Burn Unit, B-2060 Antwerp, Belgium. ${ }^{2}$ Head Clinical Unit Critical Care, Edendale Hospital, Pietermaritzburg, South Africa. ${ }^{3}$ South African Peri-operative Research Group, Discipline of Anaesthesia and Critical Care, Nelson R Mandela School of Medicine, University of KwaZulu-Natal, Durban, South Africa.

\section{Published online: 16 March 2016}

\section{References}

1. Kirkpatrick AW, Roberts DJ, De Waele J, et al. Intra-abdominal hypertension and the abdominal compartment syndrome: updated consensus definitions and clinical practice guidelines from the World Society of the Abdominal Compartment Syndrome. Intensive Care Med. 2013;39:1190-206.

2. Malbrain MLNG, Roberts DJ, De Laet I. The role of abdominal compliance, the neglected parameter in critically ill patients - a consensus review of 16 . Part 1: Definitions and pathophysiology. Anaesthesiol Intensive Ther. 2014:46:392-405.

3. Malbrain MLNG, De Laet I, De Waele J. The role of abdominal compliance, the neglected parameter in critically ill patients - a consensus review of 16 . Part 2: Measurement techniques and management recommendations. Anaesthesiol Intensive Ther. 2014;46:406-32.

4. Blaser AR, Bjorck M, De Keulenaer B, Regli A. Abdominal compliance: a bench-to-bedside review. J Trauma Acute Care Surg. 2015;78:1044-53.

5. Malbrain ML, Chiumello D, Pelosi $P$, et al. Incidence and prognosis of intraabdominal hypertension in a mixed population of critically ill patients: a multiple-center epidemiological study. Crit Care Med. 2005;33:315-22.

6. Mulier JP, Dillemans B, Heremans L. Determinants of the abdominal pressure volume relation in non ACS patients. Acta Clin Belg Suppl. 2007;62:289.

7. Mulier J, Dillemans B, Crombach M, Missant C, Sels A. On the abdominal pressure volume relationship. Internet J Anesthesiol. 2008;21:1.

8. Song C, Alijani A, Frank T, Hanna GB, Cuschieri A. Mechanical properties of the human abdominal wall measured in vivo during insufflation for laparoscopic surgery. Surg Endosc. 2006;20:987-90

9. Vlot J, Wijnen R, Stolker RJ, Bax KN. Optimizing working space in laparoscopy: CT measurement of the effect of pre-stretching of the abdominal wall in a porcine model. Surg Endosc. 2014;28:841-6.

10. Accarino A, Perez F, Azpiroz F, Quiroga S, Malagelada JR. Abdominal distention results from caudo-ventral redistribution of contents. Gastroenterology. 2009;136:1544-51.

11. Mulier JP, Coenegrachts K, Van de Moortele K. CT analysis of the elastic deformation and elongation of the abdominal wall during colon insufflation for virtual coloscopy. Eur J Anesth. 2008;25(S44):42 (abst).

12. Villoria A, Azpiroz F, Soldevilla A, Perez F, Malagelada JR. Abdominal accommodation: a coordinated adaptation of the abdominal wall to its content. Am J Gastroenterol. 2008;103:2807-15.

13. Abu-Rafea B, Vilos GA, Vilos AG, Hollett-Caines J, Al-Omran M. Effect of body habitus and parity on insufflated $\mathrm{CO} 2$ volume at various intraabdominal pressures during laparoscopic access in women. J Minim Invasive Gynecol. 2006:13:205-10.

14. McDougall EM, Figenshau RS, Clayman RV, Monk TG, Smith DS. Laparoscopic pneumoperitoneum: impact of body habitus. J Laparoendosc Surg. 1994;4:385-91.

15. Fischbach M, Terzic J, Laugel V, Escande B, Dangelser C, Helmstetter A. Measurement of hydrostatic intraperitoneal pressure: a useful tool for the improvement of dialysis dose prescription. Pediatr Nephrol. 2003:18:976-80.

16. Malbrain ML. Different techniques to measure intra-abdominal pressure (IAP): time for a critical re-appraisal. Intensive Care Med. 2004;30:357-71.

17. Sugerman $H$, Windsor A, Bessos M, Wolfe L. Intra-abdominal pressure, sagittal abdominal diameter and obesity comorbidity. J Intern Med. 1997;241:71-9.

18. Malbrain ML, De Laet I, Van Regenmortel N, Schoonheydt K, Dits H. Can the abdominal perimeter be used as an accurate estimation of intra-abdominal pressure? Crit Care Med. 2009:37:316-9.

19. Guerrero-Romero F, Rodriguez-Moran M. Abdominal volume index. An anthropometry-based index for estimation of obesity is strongly related to impaired glucose tolerance and type 2 diabetes mellitus. Arch Med Res. 2003;34:428-32

20. Kirkpatrick AW, Colistro R, Laupland KB, et al. Renal arterial resistive index response to intraabdominal hypertension in a porcine model. Crit Care Med. 2007;35:207-13.

21. Forstemann T, Trzewik J, Holste J, et al. Forces and deformations of the abdominal wall - a mechanical and geometrical approach to the linea alba. J Biomech. 2011;44:600-6.

22. van Ramshorst GH, Salih M, Hop WC, et al. Noninvasive assessment of intraabdominal pressure by measurement of abdominal wall tension. J Surg Res. 2011;171:240-4.

23. Gilroy Jr RJ, Lavietes MH, Loring SH, Mangura BT. Mead J (1985) Respiratory mechanical effects of abdominal distension. J Appl Physiol. 1985;58:1997-2003 
24. Malbrain ML, Wilmer A. The polycompartment syndrome: towards an understanding of the interactions between different compartments! Intensive Care Med. 2007;33:1869-72.

25. Malbrain MLNG, Roberts DJ, Sugrue M, et al. The polycompartment syndrome: a concise state-of-the-art review. Anaesthesiol Intensive Ther. 2014:46:433-50.

26. Wauters J, Wilmer A, Valenza F. Abdomino-thoracic transmission during ACS: facts and figures. Acta Clin Belg Suppl. 2007;62:200-5.

27. Wauters J, Claus P, Brosens N, et al. Relationship between abdominal pressure, pulmonary compliance, and cardiac preload in a porcine model. Crit Care Res Pract. 2012;2012:763181.

28. Sturini E, Saporito A, Sugrue M, Parr MJ, Bishop G, Braschi A. Respiratory variation of intra-abdominal pressure: indirect indicator of abdominal compliance? Intensive Care Med. 2008;34:1632-7.

29. De Keulenaer BL, De Waele JJ, Powell B, Malbrain ML. What is normal intraabdominal pressure and how is it affected by positioning, body mass and positive end-expiratory pressure? Intensive Care Med. 2009;35:969-76.

30. Lambert DM, Marceau S, Forse RA. Intra-abdominal pressure in the morbidly obese. Obes Surg. 2005;15:1225-32.

31. Nguyen NT, Wolfe BM. The physiologic effects of pneumoperitoneum in the morbidly obese. Ann Surg. 2005;241:219-26.

32. Sugerman HJ. Increased intra-abdominal pressure in obesity. Int J Obes Relat Metab Disord. 1998;22:1138.

33. Verbeke K, Casier I, Van Acker B, Dillemans B, Mulier J. Impact of laparoscopy on the abdominal compliance is determined by the duration of the pneumoperitoneum the number of gravidity and the existence of a previous laparoscopy or laparotomy. Eur J Anesth. 2010;27:29-30.

34. Becker V, Schmid RM, Umgelter A. Comparison of a new device for the continuous intra-gastric measurement of intra-abdominal pressure (CiMon) with direct intra-peritoneal measurements in cirrhotic patients during paracentesis. Intensive Care Med. 2009;35:948-52.

35. Cheatham ML, Malbrain ML, Kirkpatrick A, et al. Results from the international conference of experts on intra-abdominal hypertension and abdominal compartment syndrome. II. Recommendations. Intensive Care Med. 2007;33:951-62.

36. De Laet I, Malbrain ML. ICU management of the patient with intraabdominal hypertension: what to do, when and to whom? Acta Clin Belg. 2007;62(Suppl):190-9.

37. Sugerman $H$, Windsor A, Bessos M, Kellum J, Reines $H$, DeMaria E. Effects of surgically induced weight loss on urinary bladder pressure, sagittal abdominal diameter and obesity co-morbidity. Int J Obes Relat Metab Disord. 1998;22:230-5.

38. Cheatham ML, Safcsak K. Percutaneous catheter decompression in the treatment of elevated intraabdominal pressure. Chest. 2011;140:1428-35.

39. Cordemans C, De Laet I, Van Regenmortel N, et al. Fluid management in critically ill patients: the role of extravascular lung water, abdominal hypertension, capillary leak and fluid balance. Ann Intensive Care. 2012;2 Suppl 1:S1.

40. Cordemans C, De Laet I, Van Regenmortel N, et al. Aiming for a negative fluid balance in patients with acute lung injury and increased intraabdominal pressure: a pilot study looking at the effects of PAL-treatment. Ann Intensive Care. 2012;2 Suppl 1:15.

41. Hakobyan RV, Mkhoyan GG. Epidural analgesia decreases intraabdominal pressure in postoperative patients with primary intra-abdominal hypertension. Acta Clin Belg. 2008;63:86-92.

42. Tsoutsos D, Rodopoulou S, Keramidas E, Lagios M, Stamatopoulos K loannovich J. Early escharotomy as a measure to reduce intraabdominal hypertension in full-thickness burns of the thoracic and abdominal area. World J Surg. 2003;27:1323-8.

43. Oda J, Ueyama M, Yamashita K, et al. Effects of escharotomy as abdominal decompression on cardiopulmonary function and visceral perfusion in abdominal compartment syndrome with burn patients. J Trauma. 2005;59:369-74

44. Bloomfield GL, Ridings PC, Blocher CR, Marmarou A, Sugerman HJ. A proposed relationship between increased intra-abdominal, intrathoracic, and intracranial pressure. Crit Care Med. 1997;25:496-503.

45. Mulier JP, Dillemans B, Van Cauwenberge S. Impact of the patient's body position on the intraabdominal workspace during laparoscopic surgery. Surg Endosc. 2010;24:1398-402

46. Malbrain ML, Marik PE, Witters I, et al. Fluid overload, de-resuscitation, and outcomes in critically ill or injured patients: a systematic review with suggestions for clinical practice. Anaesthesiol Intensive Ther. 2014;46:361-80.
47. Voss M, Pinheiro J, Reynolds J, et al. Endoscopic components separation for abdominal compartment syndrome. Am J Surg. 2003;186:158-63.

48. Leppaniemi A, Hienonen P, Mentula P, Kemppainen E. Subcutaneous linea alba fasciotomy, does it really work? Am Surg. 2011;77:99-102.

49. De Waele JJ, Hoste EA, Malbrain ML. Decompressive laparotomy for abdominal compartment syndrome - a critical analysis. Crit Care. 2006;10:R51. 